\title{
Contradiction of the DENSITY MATRIX Notion in Quantum Mechanics
}

\author{
Ignatovich V.K. \\ Frank Laboratory of Neutron Physics, Joint Institute for Nuclear Research, Dubna, Moscow Region, Russia
}

*Corresponding Author: v.ignatovi@gmail.com

Copyright (C)2013 Horizon Research Publishing All rights reserved.

\begin{abstract}
It is shown for the first time that description of an unpolarized neutron beam by density matrix can be contradictory. Density matrix is invariant with respect to choice of a quantization axis, while experimental devices can discriminate between different quantization axes. An experiment is proposed to discriminate a direction of a quantization axis of a specially prepared nonpolarized beam. The discrimination can be seen by observation of oscillations in statistical uncertainties. The success of the experiment will mean that an entanglement, following from the EPR paradox permits communication with a superluminal speed. A criticism of the EPR paradox and of some experiments on proof of Bell's inequalities violation is presented.
\end{abstract}

Keywords Quantum Mechanics, Density Matrix, EPR Paradox

\begin{abstract}
- The statement in the title is evidently wrong at the modern level of knowledge. The density matrix is not an auxiliary construction but a result of basic concepts of quantum mechanics. More over experience with this notion is huge and convincing. As for the given paper, no doubt it contains an error. It is in inexactitude of wordings and reasonings. The problem is only how to find this error. However it is a task for the author.
\end{abstract}

Referee of JETP Lett.

\section{Introduction}

The main notions in nonrelativistic quantum mechanics are the Schrödinger equation and wave function $|\psi\rangle$, which describes so called pure state. In consideration of a beam of a particles that can stay in, say, two different states $\left|\psi_{1}\right\rangle$ and $\left|\psi_{2}\right\rangle$ with probabilities $w_{1,2}$ respectively, one uses so called density matrix

$$
\rho=w_{1}\left|\psi_{1}\right\rangle\left\langle\psi_{1}\left|+w_{2}\right| \psi_{2}\right\rangle\left\langle\psi_{2}\right|,
$$

which describes a mixed state.

In fact the density matrix can describe pure states too. It happens, in particular, when probability, say, $w_{2}$ in Eq. (1) is equal to zero. For example, if we have a beam of monochromatic polarized particles, all of them in a pure state $|u\rangle$, we can describe them with the density matrix of the form

$$
\rho=|u\rangle\langle u|
$$

The state $|u\rangle$ corresponds to polarization along some direction. If we choose the direction, which is called quantization axis, along a unit vector $\boldsymbol{a}$, the Eq. (2) is better to be represented as

$$
\rho=|\boldsymbol{a}\rangle\langle\boldsymbol{a}|,
$$

Now the question arises: how to describe a nonpolarized beam? Usually a monochromatic nonpolarized neutron beam can be described by the density matrix

$$
\rho=\frac{1}{2}(|\boldsymbol{a}\rangle\langle\boldsymbol{a}|+|-\boldsymbol{a}\rangle\langle-\boldsymbol{a}|),
$$

which shows that half of particles are polarized along some direction $\boldsymbol{a}$, and half are polarized opposite this direction. However, which direction should we choose, if we have a beam with really nonpolarized particles? Quantum mechanics tells, that it does not matter, which direction i.e. quantization axis we do choose, because Eq. (4) represents half of the unit matrix for any quantization axis, i.e. the density matrix Eq. (4) is invariant with respect to change of quantization axis. Indeed, if one chooses the quantization axis along some other unit vector $\boldsymbol{b}$, then because of expansions

$$
|\boldsymbol{a}\rangle=\alpha|\boldsymbol{b}\rangle+\beta|-\boldsymbol{b}\rangle, \quad|-\boldsymbol{a}\rangle=\alpha^{*}|-\boldsymbol{b}\rangle-\beta^{*}|\boldsymbol{b}\rangle,
$$

where $|\alpha|^{2}+|\beta|^{2}=1$, one obtains that the matrix Eq. (4) becomes

$$
\rho=\frac{1}{2}\left([\alpha|\boldsymbol{b}\rangle+\beta|-\boldsymbol{b}\rangle]\left[\alpha^{*}\langle\boldsymbol{b}|+\beta^{*}\langle-\boldsymbol{b}|\right]+\right.
$$




$$
\begin{gathered}
\left.+\left[\beta^{*}|\boldsymbol{b}\rangle-\alpha^{*}|-\boldsymbol{b}\rangle\right][\beta\langle\boldsymbol{b}|-\alpha\langle-\boldsymbol{b}|]\right)= \\
=\frac{1}{2}(|\boldsymbol{b}\rangle\langle\boldsymbol{b}|+|-\boldsymbol{b}\rangle\langle-\boldsymbol{b}|) .
\end{gathered}
$$

For instance, if $\boldsymbol{a}$ is along $y$ axis, and $\boldsymbol{b}$ is along $z$-axis, one has

$$
\begin{gathered}
|y\rangle=\frac{1}{\sqrt{2}}\left(\begin{array}{l}
1 \\
i
\end{array}\right)=\frac{1}{\sqrt{2}}(|z\rangle+i|-z\rangle), \\
|-y\rangle=\frac{1}{\sqrt{2}}\left(\begin{array}{l}
i \\
1
\end{array}\right)=\frac{1}{\sqrt{2}}[|-z\rangle+i|z\rangle],
\end{gathered}
$$

and

$\rho=\frac{1}{2}(|+z\rangle\langle+z|+|-z\rangle\langle-z|) \equiv \frac{1}{2}(|+y\rangle\langle+y|+|-y\rangle\langle-y|)$.

So two axes are equivalent for the density matrix. It will be a real scandal for quantum mechanics and mathematics, if we show that we can experimentally determine direction of quantization axis of a nonpolarized beam. It will mean that the density matrix is an artificial construction in quantum mechanics, which can be contradictory. We will consider the simplest case of a density matrix, describing a monochromatic neutron beam and propose an experiment in which we can find the direction of quantization axis in specially prepared nonpolarized neutron beam. Success of such an experiment will not only improve our understanding of quantum mechanics, but also save the science from a cancer tumor, created by EPR paradox and speculations around it.

A the end of the paper we give some criticism of the EPR paradox and physics related to it.

\section{Experimental scheme}

To achieve the goal let's consider how to prepare an unpolarized neutron beam with a fixed quantization axis and how to check experimentally the direction of the axis.

\subsection{Preparation of unpolarized neutron beam with a fixed axis}

Usually all the neutron sources produce nonpolarized neutrons. Such neutrons can be considered as particles with isotropic distribution of their spin arrows or particles described by the density matrix Eq. (4) with an arbitrary quantization axis or an isotropic distribution of quantization axes $\boldsymbol{a}$. To prepare non polarized beam with a fixed quantization or nonpolarization axis $\boldsymbol{a}$ let's consider an experiment schematically shown in fig.1.

The number of neutrons incident on the detector can be described by the matrix

$$
\boldsymbol{N}=N(|\boldsymbol{a}\rangle\langle\boldsymbol{a}|+|-\boldsymbol{a}\rangle\langle-\boldsymbol{a}|) .
$$

Therefore the detector countrate will be

$$
\langle\boldsymbol{a}|\boldsymbol{N}| \boldsymbol{a}\rangle+\langle-\boldsymbol{a}|\boldsymbol{N}|-\boldsymbol{a}\rangle \equiv N_{+}+N_{-}=2 N,
$$

and dispersion due to statistics will be

$$
\delta N=\sqrt{\delta^{2} N_{+}+\delta^{2} N_{-}}=\sqrt{2 N} .
$$

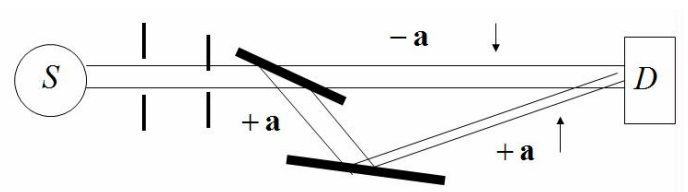

Figure 1. Scheme of production of a nonpolarized neutron beam with a fixed nonpolarization axis. A monochromatic and collimated neutron beam from a source $\mathrm{S}$ is split by a magnetic mirror into two subbeams oppositely polarized along the vector $\boldsymbol{a}$ parallel to the mirror magnetization. With the help of an additional mirror both subbeams are united at the detector D. The united beam is unpolarized one with the fixed nonpolarization axis $\boldsymbol{a}$.

\subsection{An installation to feel direction of the non- polarization axis}

To feel direction of the nonpolarization axis one needs to use a resonant spin flipper with resonant frequency $\omega$ and quantization axis along $z$, and an analyzer of polarization along $y$-axis as is shown in fig. 2 .

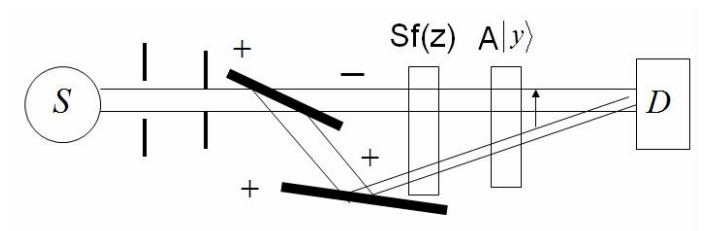

Figure 2. Modification of the scheme shown in fig.1. The two converging polarized subbeams pass through a resonant spinflipper with its axis along $z$, and through analyzer of polarization, which tr5ansmits only neutrons polarized along $y$ axis.

In this case the neutron beam with nonpolarization axis along $z$, i.e. described by the matrix Eq. (9) with $\boldsymbol{a}=(0,0,1)$ :

$$
N=N(|z\rangle\langle z|+|-z\rangle\langle-z|)
$$

will be detected with countrate $N=N_{+} / 2+N_{-} / 2$ and dispersion

$$
\delta N=\sqrt{\delta^{2}\left(\frac{N_{+}}{2}\right)+\delta^{2}\left(\frac{N_{-}}{2}\right)}=\sqrt{N} .
$$

However the neutron beam described by the matrix

$$
\boldsymbol{N}=N(|y\rangle\langle y|+|-y\rangle\langle-y|)
$$

will be detected with countrate

$$
(1+\cos (2 \omega t)) N_{+} / 2+(1-\cos (2 \omega t)) N_{-} / 2=N,
$$

and dispersion

$$
\begin{gathered}
\delta N=\sqrt{\delta^{2} N_{+} \cos ^{4}(\omega t)+\delta^{2} N_{-} \sin ^{4}(\omega t)}= \\
=\sqrt{\frac{N}{2}\left(1+\cos ^{2}(2 \omega t)\right)} .
\end{gathered}
$$

So we see that the countrates for both nonpolarization axes are equal, but dispersion is different. It is very important to check, whether it is possible to get a signal via oscillation of the dispersion. 


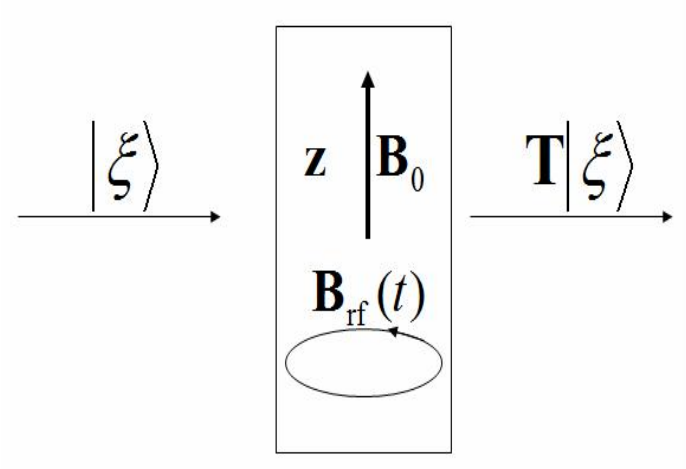

Figure 3. A resonant spin-flipper is a coil containing a permanent field $\boldsymbol{B}_{0}$ along its axis and a perpendicular to it rf field with frequency $\omega$. Transmission of a neutron in an arbitrary spin state $|\xi\rangle$ is described by a transmission matrix $\boldsymbol{T}$. The flipper, for which $\boldsymbol{T}|\xi\rangle=|-\xi\rangle$ is called $\pi$-flipper.

\subsection{The proof of the formulas}

The key element in the scheme shown in fig.2 is the resonant spin-flipper, which is frequently used in experiments on neutron optics $[1,2,3]$. Such a spin-flipper is a coil (see fig.3) with a permanent magnetic field $\boldsymbol{B}_{0}$ and perpendicular to it rotating counterclockwise rf-field

$$
\boldsymbol{B}_{r f}=b(\cos (\omega t), \sin (\omega t), 0)
$$

where $\omega=2 \mu B_{0} / \hbar$, and $\mu$ is magnetic moment of the neutron, which is aligned oppositely to the neutron spin $\boldsymbol{s}$. Direction of $\boldsymbol{B}_{0}$ can be accepted as the quantization z-axis. Interaction of neutrons with such a flipper can be solved exactly and analytically, and the solution can be explained (we consider the case of a $\pi$ flipper, in which spin of an incident particle turns to opposite direction) as follows [3].

The neutron interaction with magnetic field is described by the potential $-\boldsymbol{\mu} \cdot \boldsymbol{B}_{0}$. Therefore neutrons in the state $|z\rangle$ entering the field $\boldsymbol{B}_{0}$ are decelerated because the field in this case creates a potential barrier of the height $\mu B_{0}$.

Inside the flipper the rf-field turns the spin down, i.e. transforms the state $|z\rangle$ into $|-z\rangle$. In this state the interaction $-\boldsymbol{\mu} \cdot \boldsymbol{B}_{0}$ becomes negative, so the potential barrier transforms into potential well of depth $\mu B_{0}$. Therefore after exiting from the flipper and its magnetic field $\boldsymbol{B}_{0}$ the neutron decelerates once again. In total the neutron energy after transmission through the spin flipper decreases by the amount $2 \mu B_{0}$, which means emission of an rf quantum: $\hbar \omega=2 \mu B_{0}$. The wave functions before and after spin flipper are

$$
\begin{gathered}
\left|\psi_{\text {in }}(x, t)\right\rangle=\exp (i k x-i \Omega t)|z\rangle, \\
\left|\psi_{\text {out }}(x, t)\right\rangle=\exp \left(i k_{-}(x-D)-i(\Omega-\omega) t\right)|-z\rangle,
\end{gathered}
$$

respectively. Here $x$ is the axis of propagation, $D$ is thickness of the spin-flipper, $k$ is initial wave number, $\Omega=\hbar k^{2} / 2 m, m$ is the neutron mass, and $k_{-}=$ $\sqrt{k^{2}-2 m \omega / \hbar}$.

If the incident neutron has the state $|-z\rangle$, it accelerates, and after spin-flipper its energy becomes larger than original one by the amount $2 \mu B_{0}$, which means absorbtion of an rf quantum: $\hbar \omega=2 \mu B_{0}$. The wave functions before and after spin flipper in this case are respectively

$$
\begin{gathered}
\left|\psi_{\text {in }}(x, t)\right\rangle=\exp (i k x-i \Omega t)|-z\rangle, \\
\left|\psi_{\text {out }}(x, t)\right\rangle=\exp \left(i k_{+}(x-D)-i(\Omega+\omega) t\right)|z\rangle,
\end{gathered}
$$

where $k_{+}=\sqrt{k^{2}+2 m \omega / \hbar}$. We will not follow propagation of such spin states. We need here only to know transformation of these spin states after transmission through resonant spin flipper as shown in fig. 4 .

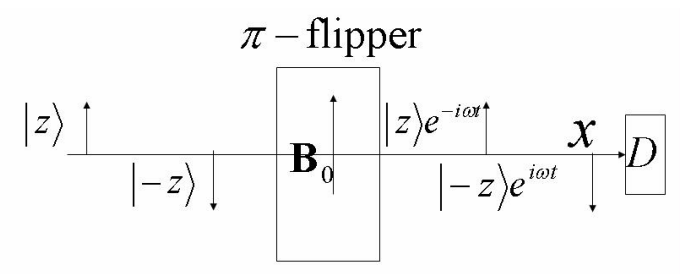

Figure 4. A neutron in the spin state $|z\rangle$ after transmission through resonant spin-flipper looses quantum of energy $\hbar \omega$ therefore it becomes in the state $|-z\rangle \exp (i \omega t)$ The incident neutron with opposite spin after transmission through resonant spin-flipper acquires energy, i.e. absorbs rf quantum and becomes in the state $|z\rangle \exp (-i \omega t)$.

If the incident neutron has a polarization $|\xi\rangle=\alpha|z\rangle+$ $\beta|-z\rangle$, its wave functions before and after spin flipper are respectively

$$
\begin{gathered}
\left|\psi_{\text {in }}(x, t)\right\rangle=\exp (i k x-i \Omega t)(\alpha|z\rangle+\beta|-z\rangle) \\
\left|\psi_{\text {out }}(x, t)\right\rangle=\alpha \exp \left(i k_{-}(x-D)-i(\Omega-\omega) t\right)|-z\rangle+ \\
+\beta \exp \left(i k_{+}(x-D)-i(\Omega+\omega) t\right)|z\rangle
\end{gathered}
$$

The spin arrow of this state represents a rotating spin wave propagating along $x$-axis.

We will not follow propagation of such a spin waves. Instead we consider two cases when initial flux is described by the matrices 1) Eq. (10) and 2) Eq. (12), which are mathematically identical, but physically can be discriminated.

In the case, when non polarization axis of the incident beam is directed along $z$ (axis of the spin-flipper), then the neutron states $| \pm z\rangle$ after transmission through spinflipper are transformed to $\exp ( \pm i \omega t)|\mp z\rangle$, and to find what flux will be at the detector after an analyzer, which transmits only states $|y\rangle$, as in the scheme of fig.2, we need to use relations

$|z\rangle=\frac{1}{\sqrt{2}}(|y\rangle-i|-y\rangle), \quad|-z\rangle=\frac{1}{\sqrt{2}}(-i|y\rangle+|-y\rangle)$.

They show that countrate of the detector will be

$$
J_{y}=N_{+}+N_{-}=N / 2+N / 2=N,
$$

and the dispersion is presented by Eq. (11).

Let's consider now the case when nonpolarization axis of the incident beam is directed along $y$-axis. According to Eq. (7) and fig.4 the state $|y\rangle$ after transmission through flipper becomes

$$
|y\rangle \rightarrow \frac{1}{\sqrt{2}}\left(e^{i \omega t}|-z\rangle+i e^{-i \omega t}|z\rangle\right),
$$


which after substitution of Eq-s (22) is transformed to

$$
|y\rangle \rightarrow \sin (\omega t)|y\rangle+\cos (\omega t)|-y\rangle
$$

Respectively

$$
|-y\rangle \rightarrow \frac{1}{\sqrt{2}}\left[e^{-i \omega t}|z\rangle+i e^{i \omega t}|-z\rangle\right],
$$

which after substitution of Eq. (22) leads to

$$
|-y\rangle \rightarrow-\sin (\omega t)|-y\rangle+\cos (\omega t)|y\rangle
$$

as shown in fig.5.

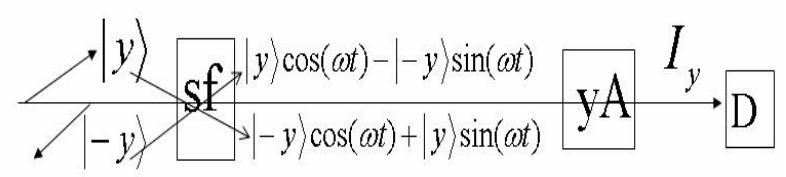

Figure 5. Transformation of spin states of two subbeams oppositely polarized along $y$-axis after transmission through a resonant spin-flipper with quantization axis along $z$.

Let's note that at $t=0$ when rf field Eq. (15) is directed along $x$-axis the transmitted spin states correspond to completely flipped $|\mp y\rangle$ states of the initial ones $| \pm y\rangle$.

Now, with the results Eq. (25) and Eq. (27) we can immediately calculate flux to detector from both polarized subbeams. If initial beam is described by the matrix Eq. (12) then the detector countrate will be

$$
J_{y}=N_{+}+N_{-}=N \sin ^{2}(\omega t)+N \cos ^{2}(\omega t)=N,
$$

However dispersion will be

$$
\delta J_{y}=\sqrt{\delta^{2} N_{+}+\delta^{2} N_{-}}=\sqrt{\frac{N}{2}\left(1+\cos ^{2}(2 \omega t)\right)},
$$

which we wanted to prove. From our consideration it follows that density matrices

$\rho=\frac{1}{2}(|y\rangle\langle y|+|-y\rangle\langle-y|), \quad \rho=\frac{1}{2}(|z\rangle\langle z|+|-z\rangle\langle-z|)$

are not equal though from mathematical point of view they are identical. We see that the notion of the density matrix in quantum mechanics is contradictory.

The main element of quantum mechanics is a wave function, and corresponding to it a pure state. If one has an ensemble of particles with different pure states, and the distribution of different states is characterized by probabilities, one must calculate a process with pure states and then average over probabilities. This is the way neutron scattering cross sections are calculated. First they are calculated for a pure state of an incident plain wave and some pure state of scatterer. Then the obtained cross section is averaged over probability distribution of the incident plain waves and over states of the scatterer. Of course the density matrix also can be useful, but because of discovered contradiction, one must be very careful with it.

\section{Consequences of the found con- tradiction}

If experiments to check a possibility to find quantization axis of the density matrix of an unpolarized particles are successful (the experiments $[4,5]$ demonstrate that they can be successful), it will mean that either EPR paradox or special relativity are wrong. Let's look at a gedanken experiment depicted in fig. 6

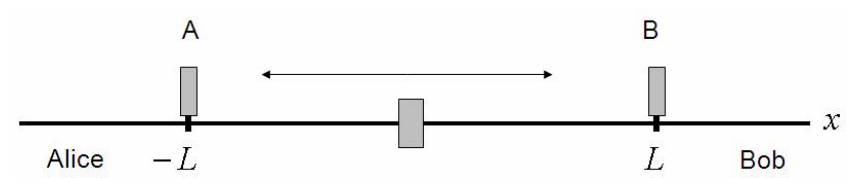

Figure 6. Scheme of a gedanken experiment for superluminal exchange information with entangled particles. Between Alice and Bob who are many light years apart, there is a source, which continuously emits toward them particles with entangled polarizations. When Bob measures polarization of particles arriving to him, he immediately defines quantization axis at Alice side. If Alice can measure direction of nonpolarization axis of particles arriving to her, Bob can change direction of his analyzer and send a message to Alice with superluminal speed.

In the experiment there is a source, which continuously emits in opposite directions couples of spin $1 / 2$ particles in singlet state. The particles are entangled, because their spin state is

$$
\Psi=\frac{1}{\sqrt{2}}(|\boldsymbol{a},-\boldsymbol{a}\rangle-|-\boldsymbol{a}, \boldsymbol{a}\rangle)
$$

where $|\boldsymbol{a}, \boldsymbol{b}\rangle$ denotes a state in which particle, going to Alice is polarized along a unit vector $\boldsymbol{a}$, and the particle, going to Bob is polarized along the unit vector $\boldsymbol{b}$. Axis $\boldsymbol{a}$ in Eq. (31) can have arbitrary direction. However, if Bob uses analyzer of polarization;, which transmits particles polarized along vector $\boldsymbol{b}$, then he immediately defines quantization axis at Alice. If his detector counts the arriving particle, then it is in the state $|\boldsymbol{b}\rangle$, and Bob knows for sure that the particle arriving to Alice, is in the state $|-\boldsymbol{b}\rangle$, and if the detector does not count the arriving particle, then it is in the state $|-\boldsymbol{b}\rangle$, and Bob knows for sure that the particle arriving to Alice, is in the state $|\boldsymbol{b}\rangle$. However Alice does not know what happens at Bob's side. The particles arriving to her are in the state described by the density matrix

$$
\rho=\frac{1}{\sqrt{2}}(|\boldsymbol{b}\rangle\langle\boldsymbol{b}|+|-\boldsymbol{b}\rangle\langle-\boldsymbol{b}|),
$$

if she cannot measure direction of the quantization axis, she can get no information from Bob. However, as we have shown, she can. Therefore Bob changing direction of his analyzer can send the signal. The signal comes immediately, but extraction of it requires some time, which depends on power of the source. In our gedanken experiment we can suppose that Alice will be able to define one position of the quantization axis during one hour. So the signal processing is slow, however it is much faster than sending signal by light, if distance $2 L$ is many light years.

The discussed possibility means that either special relativity is wrong, or there are no entangled states of far separated particles. I think that the last is true. It 
means that EPR paradox is absent, and all the physics around it, like quantum computing, quantum cryptography and quantum teleportation, is a cancer tumor on the splendid body of science. The EPR paradox is a result of wrong definitions, which led to an awful error in the original EPR paper [6]. It will be discussed in the next section.

\section{Criticism of the EPR paper [6]}

The EPR paper contains two topics: 1) the paradox itself, and 2)discussion of entangled states. I claim that neither of them exist.

\subsection{Criticism [7] of the EPR paradox}

The EPR paradox is formulated as follows [6]. The quantum mechanics is not complete and requires some additional (hidden) parameters, because it is possible to predict (with the help of entangled states) the existence of particles having simultaneously precisely defined position and momentum, while uncertainty relations prohibit it.

I claim that within quantum mechanics particles can have simultaneously precisely defined position and momentum, and uncertainty relations do not matter.

The problem of EPR emerged because of the wrong definitions of such a physical quantity as momentum $p$. According to [6] a particle's momentum is defined as an eigen value of the momentum operator $\hat{p}=-i \hbar d / d x$. Therefore the particle has a momentum only, if its state is an eigen function of the momentum operator, i.e. a plane wave:

$$
\psi(x)=\exp (i k x)
$$

where $k=p / \hbar$. With such a definition the authors of [6] claim that the relative probability to find the particle in an interval $[a b]$ is

$$
P(a, b)=\int_{a}^{b}|\psi(x)|^{2} d x=b-a .
$$

This formula is an awful error, because it writes probability in dimensional units. Such error is either not noticed or prescribed to carelessness of the Genius.

In fact the textbooks on quantum mechanics give a rule how to avoid such a defect as the one in Eq. (34). They propose to represent the plane wave in the form

$$
\psi(x)=\frac{1}{\sqrt{L}} \exp (i k x)
$$

where $L$ is some large scale. Then Eq. (34) will be modified to

$$
P(a, b)=\int_{a}^{b}|\psi(x)|^{2} d x=\frac{b-a}{L},
$$

which is dimensionless. However it is not permitted to take, for example, $b-a=2 L$, because the space is supposed to be limited by two impenetrable walls separated by the distance $L$. But in the space between the two impenetrable walls the wave function is not a plane wave. It is a function of the type

$$
\psi(x)=\sin (\pi n x / L)
$$

which is not an eigen function of the momentum operator.

So we see, if we use EPR definition of the value of the physical quantity momentum, we cannot even define a probability to find a particle somewhere. There is a dialectic: the claim that the particle is everywhere is equivalent to the claim that it is nowhere. To define a probability to find a particle somewhere, it is necessary to use wave packets, or, as is always stated in all the textbooks, we need to use the Hilbert space. However there are no vector in Hilbert space, which is an eigen vector of the momentum operator. Therefore no particle described by a vector from the Hilbert space can have a momentum according to definition of EPR. The dilemma had emerged from the incorrect definition. If we change the definition the paradox disappears.

Indeed, let's define momentum and position of a particle as

$$
p=\int d x \psi^{*} \hat{p} \psi, \quad q=\int d x \psi^{*} \hat{q} \psi
$$

where $\hat{q}$ is position operator, and $\psi$ is the wave function from Hilbert space. With such a definition the momentum and position are defined simultaneously and uncertainty relations do not matter. Therefore paradox disappears. Of course with definition Eq. (38) there also exist dispersions $\Delta p$ and $\Delta q$. However these dispersions are the properties of the Hilbert space vectors, and they can be considered as additional parameters in quantum mechanics like the frequently used notion of coherence length, which is equivalent to size of the wave packet. Such a dispersion can be defined even in classical physics for every extended in space object.

\subsection{Criticism [8] of the entangled states}

Besides this error Eq. (34) the paper, when discussing the common wave function of two separated particles, contains another inconsistency. According to EPR [6] two separated particles have a common wave function $\Psi\left(x_{1}, x_{2}\right)$, which can be expanded over complete set of eigen functions $u_{n}\left(x_{1}\right)$ of some operator, related to particle 1:

$$
\Psi\left(x_{1}, x_{2}\right)=\sum_{n} A_{n}\left(x_{2}\right) u_{n}\left(x_{1}\right)
$$

where $A_{n}\left(x_{2}\right)$ are expansion coefficients. This expansion is known as entangled state. From this expansion it follows that, if measurements with particle 1 reveals it in the state $u_{m}\left(x_{1}\right)$, then particle 2 must be in the state $A_{m}\left(x_{2}\right)$. However the particle 2 is far away from 1 , and measurements with 1 , should not affect particle 2 . Therefore particle 2 should be in the state $A_{m}\left(x_{2}\right)$ before measurements with 1 . Nobody notices that from such a conclusion it follows that the Eq. (39) must reduce to the single product $A_{m}\left(x_{2}\right) u_{m}\left(x_{1}\right)$ before the measurements. So the sum in Eq. (39) is only a list of possible products, and the measurement only reveals which product is realized in a given event of story of two particles flying apart after interaction.

Since the sum Eq. (39) is reduced to a single product, the entangled state does not exist. But this conclusion is not the end of the story. If the wave functions of separated particles belong to the Hilbert space and are 
represented by wave packets, their products annuls with time. Therefore the common wave function of separated particles, if unimportant motion of the center mass is ignored, should be represented not by a product, but by a sum of functions, or by a single function of coordinates difference: $f\left(x_{1}-x_{2}\right)$, which is also a vector of Hilbert space, i.e. goes to zero when $\left|x_{1}-x_{2}\right| \rightarrow \infty$.

\subsection{Criticism [9] of the Bohm-Aharonov ap- proach to the EPR paradox}

Bohm-Aharonov [10] considered EPR paradox and entanglement in terms of spin operators of $1 / 2$ spin particles, and photon's polarizations. We will show that there are neither paradox, nor entanglement. For the sake of brevity we consider only spin $1 / 2$ particles.

In the paper [10] it is erroneously written that

In quantum theory, a difficulty arises, in the interpretation of the above experiment, because only one component of the spin of each particle can have a definite value at a given time. Thus, if the $\mathrm{x}$ component is definite, then $\mathrm{y}$ and $\mathrm{z}$ components are indeterminate and we may regard them more or less as in a kind of random fluctuation.

It is absolutely incorrect, Every normalized spinor $|\psi\rangle$ describes a particle absolutely precisely polarized along some unit classical vector $\boldsymbol{a}$ (let's call it "spin arrow") defined as

$$
\boldsymbol{a}=\langle\psi|\boldsymbol{\sigma}| \psi\rangle,
$$

where $\boldsymbol{\sigma}=\left(\sigma_{x}, \sigma_{y}, \sigma_{z}\right)$ are three Pauli matrices. The spin arrow has all the components precisely defined. If one knows direction of the vector $\boldsymbol{a}$ and aligns axis of his analyzer along $\boldsymbol{a}$, he will register the particle with unit probability.If there were some fluctuating components the spin would never have the fixed value. All the experimentalists working with polarized beams know that there are no fluctuating spin components perpendicular to the direction of the beam polarization.

Further the authors write:

we could say that on measuring the $z$ component of the spin of particle, we disturb the $x$ and $y$ components and make them fluctuate.

This is not a correct description of measurements. A measurement is an active filtering process, which transforms the state of the incident particle, and the transformed state has no fluctuating spin components. For instance, if a beam of particles polarized in $x$ direction is sent to an analyzer with its axis along $z$ direction, the beam is transformed into two sub beams. The one, transformed with the operator $\left(I+\sigma_{z}\right) / 2(I$ is the unit matrix), passes through the filter and becomes polarized along $z$ axis. The other sub beam, transformed with the operator $\left(I-\sigma_{z}\right) / 2$, reflects from the filter and becomes polarized along $-z$ direction. One can see that there are no fluctuations in $x$ and $y$ components of spin polarization in both sub beams.

One important contribution of the paper [10] is introduction of the entangled spin states of two particles shown in Eq. (31). However spin state of a particle is accompanied by coordinate part $\psi(x)$, and if $\psi(x)$ is a vector of Hilbert space, then, as we discussed above, the product of two functions for separated particles goes to zero with time. Therefore entangled states in the form Eq. (31) cannot exist.

\subsection{Short criticism of the experiments on demonstration of violation of Bell's in- equalities [11]}

All my criticism of the EPR paradox is met with scepticism, since there are so many performed experiments, which demonstrated violation of Bell's inequality, and the violation is considered as a proof of the entanglement. However all these experiments are biased by desire of experimentalists to prove the entanglement. In most of the experiments a correlation between polarizations of two separated photons is measured in coincidence. This correlation is different in the case of entanglement and in the case of absence of the entanglement. However the difference is a constant, which is not present in the case of entanglement. This constant in experiments can be excluded as a background [12], and then we are left with correlations proving the entanglement.

More over, results of the experiments depend on tuning of the coincidence events, and this tuning can be arranged in such a way that one can get not only violation, but even super violation of the Bell's inequalities, as is shown in [13]. There are a lot of references, which show different defects of the conducted experiments, and at present there is an opinion that still no experiment was made without a loophole. I suppose that proposed here the feasible experiment proving contradiction of the density matrix will stop the useless search for loophole free experiment to prove entanglement of far separated particles.

\section{Conclusions}

I considered an experiment, which, in the case of positive result, will prove that the density matrix is a contradictory notion in quantum mechanics. An unpolarized beam of spin $1 / 2$ particles can be prepared as an aligned beam with a fixed quantization axis, and the axis can be measured, though mathematics proves that such a beam is invariant with respect to change of the quantization axis, and therefore the axis cannot be determined. Success of the experiment will also prove that the entanglement of polarizations of separated particles can be used for transmission of a signal with a superluminal velocity. Such a possibility means that special relativity or entanglement are wrong. I think that wrong is the entanglement, which stemmed from EPR paradox. The paradox itself is a consequence of the erroneous definition of physical quantities in the EPR paper [6]. It is surprising that so many scientist were unable to pinpoint the error. I discussed this error, the science aimed at proving the entanglement, and I had shown that from the very logic of the EPR paper [6] it follows that there are no entanglement at all of the far separated particles in quantum mechanics. 


\section{REFERENCES}

[1] Vladimir K. Ignatovich and Filipp V. Ignatovich The Kruger problem and neutron spin games. Am. J. Phys., v. 71, pp. 1013-1024, 2003.

[2] R.Gahler, V.K.Ignatovich, Neutron holography without reference beams, Physics letters A, v. 362, pp 393-400, 2007.

[3] M. Utsuro, V. K. Ignatovich, Handbook of Neutron Optics, (Wiley-VCH, Weinheim, 2010.

[4] Ebisawa T, Yamazaki D, Tasaki S, Kawai T, Hino M, Akiyoshi T, Achiwa N, Otake Y. Quantum beat experiments using a cold neutron spin interferometer. J.Phys.Soc.Jap. v. 67, pp. 1569-73, 1998.

[5] Achiwa N, Shirozu G, Ebisawa T, Hino M, Tasaki S, Kawai T, Yamazaki D. Time beat neutron spin interferometry before and after analyzer. J.Phys.Soc.Japan. v. 70(Suppl. A), pp. 436-8, 2001.

[6] Einstein A, Podolsky B, Rosen N. Can quantummechanical description of physical reality be considered complete? Phys. Rev. v. 47(May 15), pp.777-80, 1935.

[7] Ignatovich V.K., Einstein Podolsky Rosen paradox, Online available from http://textbookphysics.org/
[8] Ignatovich V.K., Comment On "Can QuantumMechanical Description of Physical Reality Be Considered Complete?", Online available from http://jinr.academia.edu/VladimirKIgnatovichPublications

[9] Ignatovich V.K., Comment On "Discussion of Experimental Proof for the Paradox of Einstein, Rosen, and Podolsky", Online available from http://jinr.academia.edu/VladimirKIgnatovichPublications.

[10] D. Bohm, Y.Aharonov., Discussion of experimental proof for the paradox Einstein, Rosen, and Podolsky Phys. Rev. Vol. 108, p. 1070, 1957.

[11] John S. Bell. On the EPR paradox. Physics Vol. 1, pp. 195-200, 1964.

[12] Vladimir K. Ignatovich, On epr paradox, bell's inequalities and experiments that prove nothing,Concepts of Physics, Vol. V No. 2, 1-45, 2008.

[13] Ignatovich V.K., "A Model of Violation and Superviolation of Bell's Inequality in Local Quantum Mechanics", Online available from http://jinr.academia.edu/VladimirKIgnatovichPublications 